\title{
Interesterification of a commonly consumed palm-based hard fat blend does not affect postprandial lipoprotein metabolism in healthy older adults
}

\author{
$\underline{\text { Wendy Hall }}^{1}$, Charlotte Mills ${ }_{5}^{2}$, Robert Gray ${ }^{1}$, Scott Harding $^{3}$, Barbara Fielding ${ }^{4}$, \\ Johanna Bruce ${ }^{5}$, Caroline Le Roy ${ }^{1}$ and Sarah Berry ${ }^{1}$ \\ ${ }^{1}$ King's College London, London, United Kingdom, \\ ${ }^{2}$ University of Reading, Reading, United Kingdom, \\ ${ }^{3}$ Memorial University, St John's, Canada, \\ ${ }^{4}$ University of Surrey, Guildford, United Kingdom and \\ ${ }^{5}$ ADM Oils \& Fats, Erith, United Kingdom
}

\begin{abstract}
Interesterified (IE) fats are widely used to replace partially-hydrogenated fats as hard fats with functional and sensory properties needed for spreads/margarines, baked goods, and confectionary, while avoiding the health hazards of trans fats. Detailed mechanistic work to determine the metabolic effects of interesterification of commonly-consumed hard fats has not yet been done. Earlier studies using fats less commonly consumed have shown either neutral or a lowering effect on postprandial lipaemia. We investigated postprandial lipaemia, lipoprotein remodelling, and triacylglycerol-rich lipoprotein (TRL) fraction apolipoprotein concentrations following a common IE blend of palm oil/kernel fractions versus its non-IE counterpart, alongside a reference monounsaturated (MUFA) oil. A 3-armed, double blind, randomized controlled trial (clinicaltrials.gov NCT03191513) in healthy adults ( $=20$; 10 men, 10 women) aged 45-75 y, assessed effects of single meals ( $897 \mathrm{kcal}, 50 \mathrm{~g}$ fat, $16 \mathrm{~g}$ protein, $88 \mathrm{~g}$ carbohydrate) on postprandial plasma triacylglycerol (TAG) concentrations, lipoprotein profiles, and TRL fraction apolipoprotein B48 and TAG concentrations. Test fats were IE 80:20 palm stearin/palm kernel fat, the equivalent non-IE fat, and a high-MUFA reference oil (rapeseed oil, RO). Blood was collected at baseline and hourly for $8 \mathrm{~h}$. Linear mixed modelling was performed, adjusting for treatment order and baseline values (ver. 24.0; SPSS Inc., Chicago, IL, USA). Total $8 \mathrm{~h}$ incremental area under the curves (iAUC) for plasma TAG concentrations were lower following IE and non-IE compared with RO (mean difference in iAUC: non-IE vs. RO -1.8 mmol/L.h (95\% CI -3.3, -0.2); IE vs. RO $-2.6 \mathrm{mmol} / \mathrm{L} . \mathrm{h}(95 \% \mathrm{CI}-5.3,0.0))$, but iAUCs for IE and non-IE were not significantly different. There were no differences between IE and non-IE for chylomicron fraction apoB48 concentrations nor TAG:apoB48 ratio. No differences were observed between IE and non-IE for lipoprotein (VLDL, HDL, LDL) particle size or sub-class particle concentrations. However, LDL particle diameters were reduced at 5 and $6 \mathrm{~h}$ following IE vs RO $(P<0.05)$. XXL- (including chylomicron remnants and VLDL particles), XLand L-VLDL particle concentrations (average diameters $>75,64$, and $53.6 \mathrm{~nm}$ respectively) were higher following IE and non-IE vs. $\mathrm{RO}$ at $6 \mathrm{~h}(P<0.05)$ and $8 \mathrm{~h}$ postprandially $(P<0.005-0.05)$. In conclusion, both IE and non-IE palmitic acid-rich fats generated a greater preponderance of pro-atherogenic large TRL remnant particles in the late postprandial phase relative to an oleic acid-rich oil. However, the process of interesterification did not modify postprandial TAG response or lipoprotein metabolism.
\end{abstract}

\section{Conflict of Interest}

Jo Bruce is employed by ADM, a manufacturer of edible oils and fats.

There are no other potential conflicts of interest to declare. 